\title{
Inflammatory Enlargement of the Gingiva: A Case Report
}

\author{
Nitin Tomar', Cherry Jain², Apoorva Arun², Manini \\ ${ }^{1}$ Associate Professor, Department of Periodontology, Subharti Dental College, Meerut, India, \\ ${ }^{2} P G$ Student, Department of Periodontology, Subharti Dental College, Meerut, India.
}

\begin{abstract}
Background: Gingival enlargement is one of the frequent features of gingival diseases. Inflammation of the gingival tissues can be induced by diverse method. All changes in gingival tissues express with some degree of inflammation. In some cases, where gingival enlargement could be the primary sign of potentially lethal systemic diseases, a correct diagnosis of these enlargements could prove life saving for the patient or at least initiate early treatment and improve the quality of life. Pertinent management depends on precisely diagnosing the origin of enlargement. The aim of publishing this case report is to present the clinical, histopathological features and treatment of inflammatory gingival enlargement which disturbed the aesthetics and masticatory function of the patient.
\end{abstract}

Method: A 20 year old female patient reported with a chief complaint of swelling in the gums of teeth in lower front region. The enlargement was soft and friable and bleeding spontaneously making it difficult for the patient to maintain adequate personal oral hygiene. Surgical therapy was carried out to provide a good aesthetic outcome.

Conclusion: No recurrence was reported. Gingival overgrowth is unaesthetic, and may interfere in mastication and speech; hence a thorough understanding of the pathogenesis is essential for management.

Keywords: Inflammatory, Hyperplastic, Gingiva, Enlargement, Local factors, Electrosurgery, Histopathology.

\section{Introduction}

Oral mucosa is persistently subjected to external and internal stimuli and therefore manifests a continuum of diseases that range from developmental to reactive, inflammatory to neoplastic. ${ }^{1}$ For almost four millennia the clinical manifestations of gingival diseases have been noted by mankind. It was not until the last half of the 20th century that our views about the nature of gingival diseases began to emerge,there has been growing acceptance that gingivitis does not represent a single disease but rather a spectrum of diseases that are

\footnotetext{
Corresponding Author:

Cherry Jain

Department of Periodontology, Subharti Dental

College, NH 58, Subhartipuram, Meerut 250003, India

e-mail: cherryjain06@gmail.com

Tel: +91-7618373693
}

the outcome of a variety of different processes. Although inflammation of the gingival tissues can be induced by diverse method (e.g. trauma, chemical agents, temperature extremes, ionizing radiation, viruses, fungi, immune defects, etc.), at this time gingival diseases are considered to be disease entities that are initiated by dental plaque and are restricted to gingival tissues. ${ }^{2}$

All changes in gingival tissues express with some degree of inflammation. Pertinent management depends on precisely diagnosing the origin of enlargement. However, the skills of a clinician are put to test when arriving at a particular diagnosis among the myriad of gingival enlargements that can be classified according to etiologic factors and pathologic changes, according to location and distribution and/or according to the degree of enlargement. ${ }^{3}$ Indices are important for quantification of the extent and severity of gingival overgrowth. For example, the degree of gingival enlargement can be scored asfollows: ${ }^{4}$ 
Grade 0: no signs of gingival enlargement

Grade I: enlargement confined to interdental papilla

Grade II: enlargement involves papilla and marginal gingiva

Grade III: enlargement covers three-fourths or more of the crown.

When a patient with gingival enlargement is seen, the initial assessment is made by careful visual examination of abnormalities of gingival contours, texture, and color, which are compared with normal standards. Visual inspection is accompanied by a detailed medical history to exclude potential systemic factors and conditions. An accurate assessment is critical for planning the method of therapy and the maintenance phase.

Case Report: A 20 year old female patient reported to the Department of Periodontology, Subharti Dental College and Hospital, Meerut with a chief complaint of swelling in the gums of teeth in lower front region. Local factors, like plaque and calculus were present. The gingiva seemed to be markedly enlarged - GradeIII gingival enlargement. The enlargement appeared diffused, red and spongy. The gingiva was soft and friable with a smooth, shiny appearance with probing depth of more than $5 \mathrm{~mm}$ and generalized spontaneous gingival bleeding on probing (Fig 1). There was nodrug or systemic history reported. Also, there was no familial history present. A provisional diagnosis of inflammatory gingival enlargement was made.

On the first visit, scaling and root planing were performed and the patient was counselled regarding the maintenance of her oral hygiene. Chlorhexidine 1.2\% mouthwash was prescribed and warm saline rinses were advised along with it. The patient was recalled in 14 days. On the basis of the existing enlargement, gingivectomy with electrosurgery was planned (Fig.2,3,4). A written informed consent was obtained before the surgical procedure. Residual plaque and calculus were removed followed by thorough root planing (Fig.5).

The excised tissue was sent for histopathological examination (Fig.6). Haemotoxylin and eosinstaining showed proliferation of basal junctional epithelium along with inflammatory cells and fluid, and vascular engorgement (Fig.7). The features were suggestive of inflammatory epithelial hyperplasia.
The patient was given post-operative instructions and prescribed antibiotic and anti-inflammatory drugs TDS for 5 days. Chlorhexidine mouthwash was continued twice daily for 3 weeks. Patient was recalled after 10 days for check-up (Fig.8).

The patient has been kept on maintenance since and no recurrence has been seen in subsequent follow up visits.

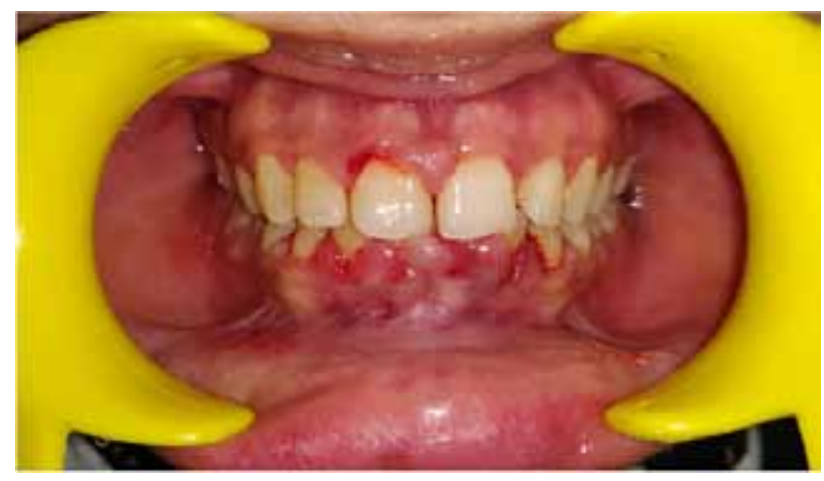

Fig. 1- Pre-op view

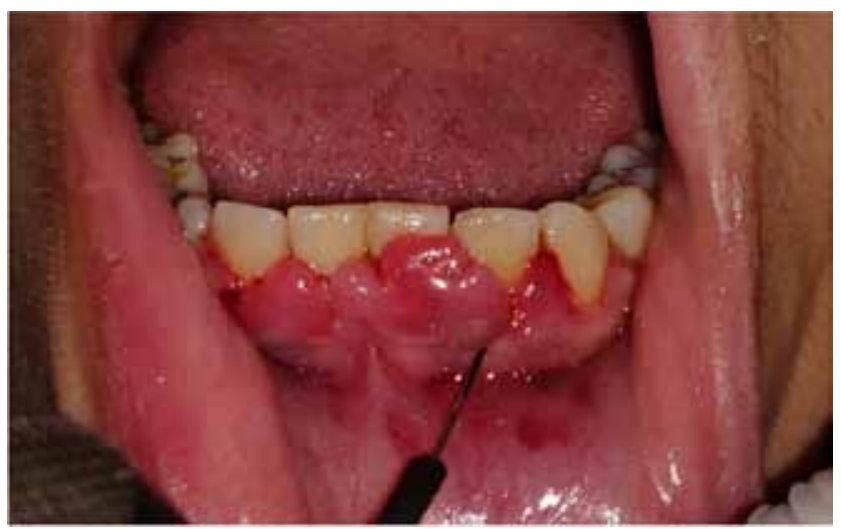

Fig. 2- Enlargement after phase 1

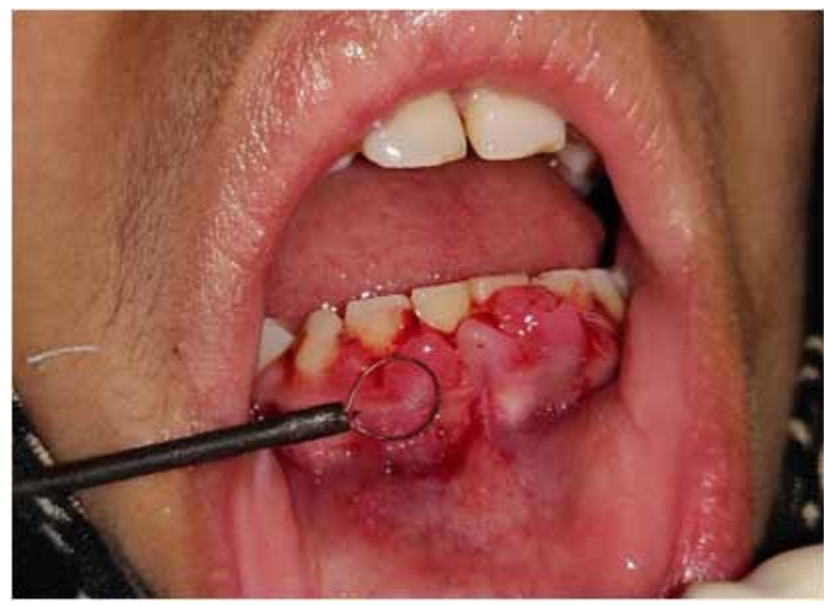

Fig. 3- Removal of enlargement with electrosugery 


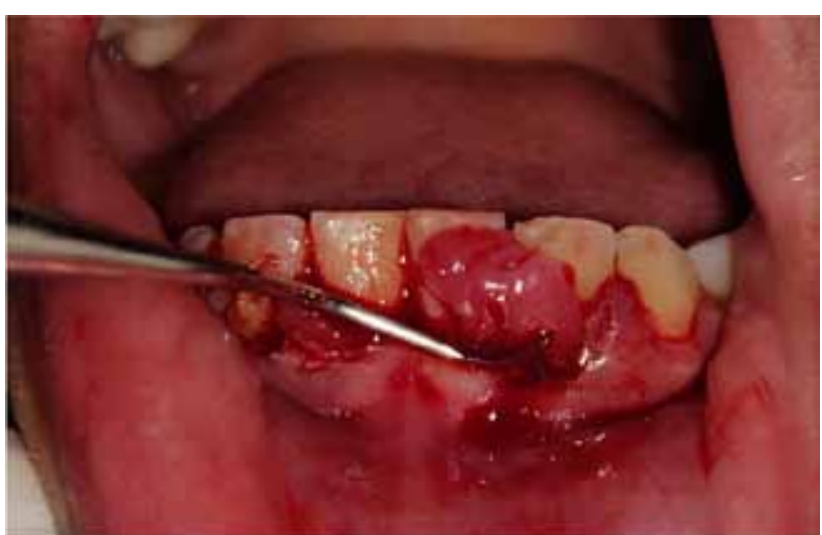

Fig. 4- Removal of inflamed tissue

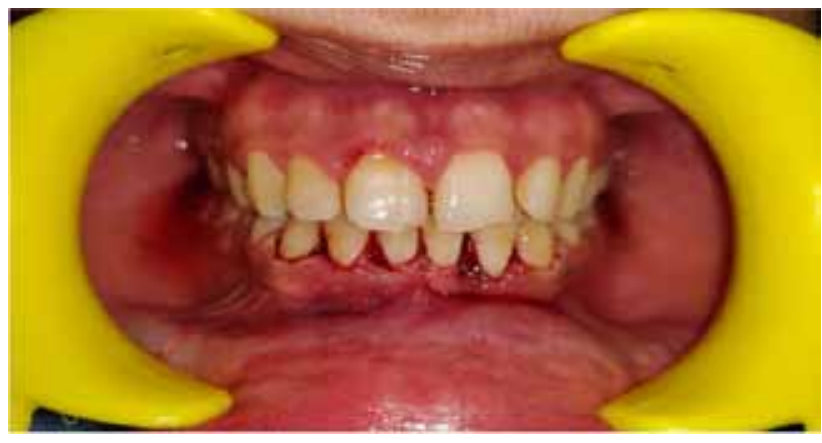

Fig.5- After removal of enlargement

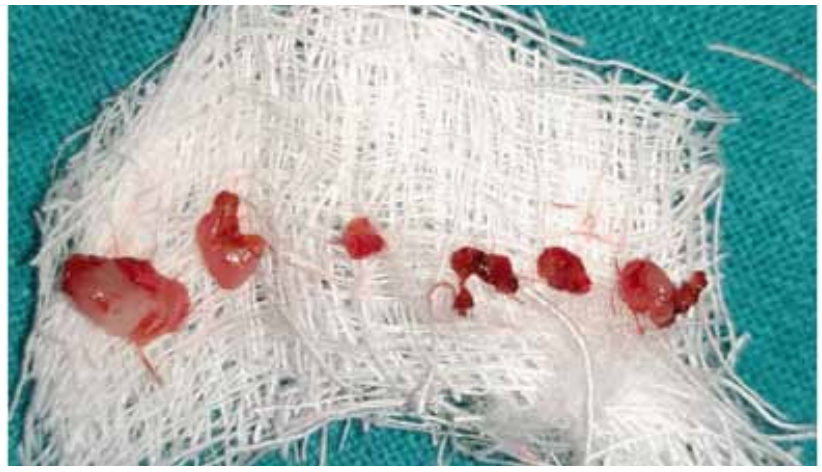

Fig. 6- Excised Tissue sent for histopathological examination

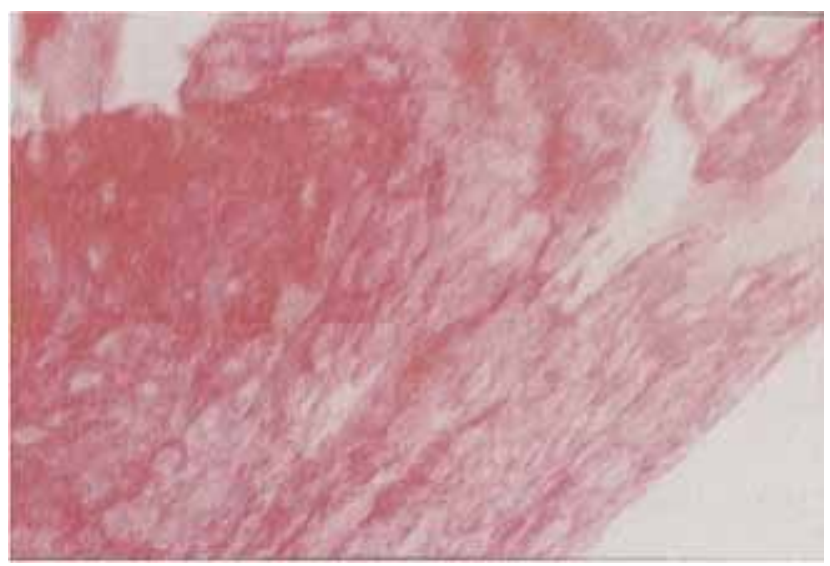

Fig. 7- Haemotoxylin and eosin staining

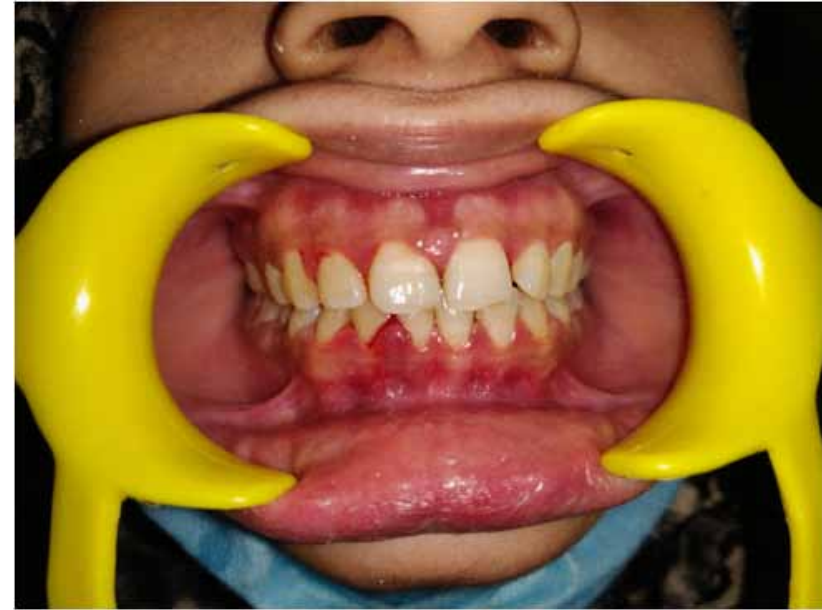

Fig. 8- 10 days post-op

\section{Discussion}

Gingival overgrowth varies from mild enlargement of isolated interdental papillae to uniform marked enlargement affecting either one or both the jaws. ${ }^{5}$ Although different types of inflammation may be features of a specific diagnosis, inflammation per se is not a diagnosis in itself. More specifically, the clinical presence of an inflammatory response should not necessarily be considered a sign of disease. ${ }^{6}$ At the other end of the spectrum, the absence of clinical signs of inflammation may not exclude the presence of an ongoing inflammatory process evident at a histologic level. For example, during cigarette smoking,the gingival inflammatory response to plaque accumulation on teeth will be muted, despite distinct gingival hostresponse patterns. ${ }^{7}$

The predilection for attachment loss at inflamed gingival sites may be dependent on the susceptibility and responsiveness of the individual to the inflammatory insult. ${ }^{8}$ Moreover, specific types of inflammatory responses in the gingiva are necessary to initiate destruction of the connective tissue attachment apical to the cemento-enamel junction. The inter-relationships between health and gingivitis and periodontitis are complex and depend upon a symbiotic or a dysbiotic biofilm and the proportionality of the host's immuneinflammatory response and its ability to resolve inflammation. ${ }^{9}$

Differential diagnosis of gingival enlargement with thorough dental and medical history, examination and identification of etiologic factors is required to make an informed decision regarding the treatment plan of the patient for a successful resolution of the condition. 


\section{Conclusion}

Gingival overgrowth is unaesthetic, and may interfere in mastication and speech. To prevent attachment loss and destruction of periodontal tissue, dealing with gingivitis and gingival enlargement by appropriate local therapeutic intervention is essential. A thorough knowledge of gingival overgrowth and the patient's systemic and oral medical histories are critical for designing the treatment and maintaining outcomes.

\section{References}

1. Effiom OA, Adeyemo WL, Soyele OO. Focal reactive lesions of the gingival: An analysis of 314 cases at tertiary health institution in Nigeria. Niger Med J 2011;52:35-40.

2. Mariotti A. Plaque-Induced Gingival Diseases In: Lang NP, Lindhe J editors Clinical Periodontology and Implant Dentistry 6th ed. U.K.: John Wiley \& Sons, Ltd; 2015. pp. 366-380.

3. Agrawal AA. Gingival enlargements: Differential diagnosis and review of literature. World J Clin Cases 2015;3(9):779-788.
4. Kantarci A, Carranza FA, Hogan E. Gingival Enlargement In: Newman MG, Takei HH, Klokkevold PR, Carranza FA editors Newman andCarranza'sClinicalPeriodontology 13th ed. Philadelphia : Elsevier 2019. pp. 1523-1572.

5. Tomar N, Jain G, Sharma A, Wadhawan A. Inflammatory Gingival Enlargement - A Case Report. Int J Oral Health Dent 2015;1(3):146-148.

6. Murakami S, Mealey BL, Mariotti A, Chapple ILC. Dental plaque-induced gingival conditions. J Clin Periodontol 2018;45(20):17-27.

7. Bergstrom J, Preber H. The influence of cigarette smoking onthe development of experimental gingivitis. J Periodontal Res1986;21:688-676.

8. Chapple IL, Bouchard P, Cagetti MG. Interaction of lifestyle,behaviour or systemic diseases with dental caries and periodontal diseases: Consensus report of group 2 of the joint EFP/ORCAworkshop on the boundaries between caries and periodontal diseases. J Clin Periodontol. 2017;44(18):39-51.

9. Meyle J, Chapple I. Molecular aspects of the pathogenesis of periodontitis. Periodontol 2000. $2015 ; 69: 7-17$. 